\title{
Importanza della pulsatilità della PRL per la diagnosi di iperprolattinemia
}

\author{
Salvatore Cannavò ${ }^{1}$
}

Pubblicato online: 14 luglio 2015

(C) Springer International Publishing AG 2015

\section{Commento a:}

\section{Importance of cannulated prolactin test in the definition} of hyperprolactinaemia.

M.B. Whyte, J.A. Gilbert, A.M. McGregor, S. Pramodh, J.P. Miell, L. Srikugan, R.A. Sherwood, S.J.B. Aylwin. Pituitary (2015) 18:319-325

Il lavoro di Whyte et al. tratta un argomento di grande attualità per gli endocrinologi, da quando le linee guida pubblicate nel 2006 su Clinical Endocrinology [1] e nel 2011 sul Journal of Clinical Endocrinology and Metabolism [2] hanno affermato che il singolo dosaggio di PRL è sufficiente per diagnosticare l'iperprolattinemia in un appropriato contesto clinico, mentre il prelievo seriato, impropriamente indicato come pulsatilità, è necessario solo nei casi dubbi, ovvero quando l'iperprolattinemia non è sintomatica e non risulta causata da un eccesso di macroprolattina.

In questo studio gli autori hanno retrospettivamente esaminato i risultati dei prelievi seriati effettuati durante un decennio (2000-2010) in 235 pazienti con iperprolattinemia determinata su singolo prelievo, che non era attribuibile a macroprolattinemia, insufficienza renale, insufficienza epatica, ipotiroidismo, assunzione di farmaci o gravidanza. La prima determinazione di PRL, che aveva portato alle ulteriori indagini, è stata definita referral prolactin. Tutti i pazienti sono stati sottoposti a rivalutazione dei livelli di PRL sul prelievo eseguito al momento del posizionamento di un catetere venoso, definiti repeat prolactin, e su un prelievo eseguito dallo stesso catetere 120 minuti dopo, indicati come resting prolactin. Complessivamente, la condizione di iperprolattinemia è stata confermata nel 74\% dei casi, men-

S. Cannavò

cannavos@unime.it

1 UOC di Endocrinologia, AOU Policlinico G. Martino, Via Consolare Valeria, 1, 98125 Messina, Italia tre i valori di repeat prolactin o di resting prolactin sono risultati normali nel 17 e nel $9 \%$ dei casi, rispettivamente. Il livello medio di referral prolactin è risultato maggiore, anche se non significativamente, nei pazienti con iperprolattinemia definitivamente confermata (iperprolattinemia vera) rispetto a coloro in cui la prolattina è risultata normale in occasione delle successive valutazioni (sia repeat prolactin che resting prolactin). La risonanza magnetica ipofisaria, che è stata eseguita nell' $85 \%$ dei pazienti con iperprolattinemia vera, ha dimostrato la presenza di alterazioni morfologiche nel $65 \%$ dei casi. Il valore più elevato di referral $P R L$ nel gruppo di pazienti con normale resting prolactin è stato pari a $53 \mathrm{ng} / \mathrm{ml}$, mentre il valore di referral prolactin indicativo di iperprolattinemia vera, calcolato mediante curva ROC, è risultato pari a $96,2 \mathrm{ng} / \mathrm{ml}(4 \times \mathrm{ULN})(\mathrm{ULN}=$ limite superiore del valore di riferimento), con specificità del $97 \%$. Per quanto riguarda il contesto clinico, la galattorrea è risultata l'unico segno significativamente più frequente nelle donne con iperprolattinemia vera, mentre i livelli di testosterone sono risultati significativamente più bassi nei maschi con iperprolattina vera rispetto agli altri.

In conclusione, lo studio di Whyte et al. dimostra che un livello random di PRL $>4 \times$ ULN è indicativo di una condizione di iperprolattinemia vera con una specificità del 97\%, mentre la determinazione dei livelli di PRL mediante prelievo seriato a riposo (pulsatilità) è indicata nei pazienti con valori random di PRL superiori a $50 \mathrm{ng} / \mathrm{ml}$.

\section{Bibliografia}

1. Casanueva FF, Molitch ME, Schlechte JA et al (2006) Guidelines of the Pituitary Society for the diagnosis and management of prolactinomas. Clin Endocrinol 65:265-273

2. Melmed S, Casanueva FF, Hoffman AR et al (2011) Endocrine Society. Diagnosis and treatment of hyperprolactinemia: an Endocrine Society clinical practice guideline. J Clin Endocrinol Metab 96:273-288 